\title{
Sequential priming of 3-D perceptual organization
}

\author{
JASON S. MCCARLEY and ZIJIANG J. HE \\ University of Louisville, Louisville, Kentucky
}

\begin{abstract}
In four experiments, the effects of sequential priming on the perceptual organization of complex three-dimensional (3-D) displays were examined. Observers were asked to view stereoscopic arrays and to search an embedded subset of items for an odd-colored target while 3-D orientation of the stimuli was varied randomly between trials. Search times decreased reliably when 3-D stimulus orientation was unchanged on consecutive trials, indicating substantial sequential priming by 3-D stimulus layout. The priming was nonsensory and was independent of priming by additional stimulus characteristics. Finally, priming by 3-D layout was unaffected by observers' foreknowledge of display orientation. Results indicate that perceptual organization of 3-D stimuli is guided by a short-term trace of 3-D spatial relationships between stimuli.
\end{abstract}

The visual world is characterized by redundancy. Visual stimuli rarely change randomly from one moment or place to the next, but vary across space and time in largely predictable ways. Realizing this, Attneave (1954) and Barlow (1961) suggested that a primary aim of perceptual encoding might be to exploit this regularity. By recognizing and efficiently encoding behaviorally relevant patterns, the visual system could expunge much of the redundancy within the raw visual array and increase the economy with which vital information is represented. Attneave suggested that the phenomena of perceptual organization (Wertheimer, 1923/1958) might emerge from this tendency toward economical representation. Barlow proposed that representation would be maximally efficient if the most pervasive and behaviorally germane regularities of the visual world were most readily encoded.

Some evidence demonstrating that visual representation is indeed guided by this principle has shown that stimuli are perceptually organized with greater ease when they more closely mimic the behaviorally relevant structure of the observer's visual environment, even when displays are otherwise matched in complexity. A ubiquitous and important feature of the human observer's natural visual ecology, for example, is the underlying surface of support, which ensures that surfaces and objects relevant to human behavior tend to lie farther in depth with increasing height in the field (Gibson, 1950; Sedgwick, 1986). Objects distributed across the ground, tabletops,

J.S.M. is now at the Beckman Institute for Advanced Science and Technology, University of Illinois. This research was supported by Sigma Xi Grant-in-Aid of Research to J.S.M. and by a Sloan Research Fellowship and a grant from the College of Arts and Sciences from the University of Louisville to Z.J.H., and by Grant ONR 994984. Some of the results were presented at the 1998 meeting of the ARVO. The authors thank G. J. Andersen, T. Sanocki, and H. A. Sedgwick for their helpful comments on an earlier draft. Correspondence concerning this article should be addressed to J. S. McCarley, Beckman Institute, University of Illinois at Urbana-Champaign, 405 North Mathews Avenue, Urbana, IL 61801 (e-mail: mccarley@uiuc.edu). desktops, and so forth, rise in the field of view as they become farther in depth. Accordingly, McCarley and $\mathrm{He}$ (2000) found that observers could perceptually organize complex three-dimensional (3-D) displays most easily when stimuli were oriented top away, or ground-like, relative to the observer, rather than when they were oriented bottom away, or ceiling-like, even though the stimuli were identical in the structures (see Figures 1 and 2 below). The observers' ability to perceptually organize the stimulus displays appears to have been mediated by their experience within the natural visual ecology, throughout which the 3 -D layout objects can assume, relative to the observer's vantage point, is largely constrained.

Regularities within the structure of a visual environment, however, may exist at various spatial and temporal scales. Although some characteristics of the visual array obtain across nearly the whole of the natural visual environment, others persist only locally. The layout of surfaces immediately surrounding an observer is unlikely to change drastically or arbitrarily from moment to moment, for example, even if the details of the layout are not replicated anywhere else within the visual world. Thus, just as the visual system can achieve efficiency of representation by exploiting long-term, pervasive regularities of the larger visual environment, it might also facilitate representation by recognizing and preferentially encoding patterns that exist over smaller regions and for shorter durations; given the complexity of the natural visual world, some implicit memory for the details of particular objects and scenes might be prerequisite for adaptive visual control of behavior (Chun \& Nakayama, 2000). Over extended periods of time, such memory may be effected through long-term storage of representations of individual stimuli (e.g., Chun \& Jiang, 1998, 1999; Lassaline \& Logan, 1993). Robertson (1996) suggested that, over briefer durations, visual performance might also be facilitated by an attentional print - that is, a short-lasting trace of attentional weights assigned to the features by which the visual field is parsed into surfaces, groups, or objects. Evidence for the attentional print comes 
from the demonstration of trial-to-trial priming of selection in hierarchical patterns (Kim, Ivry, \& Robertson, 1999; Lamb, London, Pond, \& Whitt, 1998; Lamb, Pond, \& Zahir, 2000; Robertson, 1996; Robertson, Egly, Lamb, \& Kerth, 1993; Ward, 1982) and from visual search displays (Maljkovic \& Nakayama, 1994, 1996).

In the present experiments, we sought evidence that visual encoding of complex 3 -D patterns might likewise exploit redundancy of the local visual environment, as would be evinced by short-term priming of 3-D perceptual organization. Past results have indicated that priming can occur at fairly high levels of visual object representation. Researchers have found, for example, that priming in object-naming tasks can persist across translations and reflections (Biederman \& Cooper, 1991), changes in size (Biederman \& Cooper, 1992), viewpoint (Bartram, 1974; Biederman \& Gerhardstein, 1993), shading (Cave \& Squire, 1992), texture, and color (Cave, Bost, \& Cobb, 1996). Moreover, such effects have been found to persist over the course of minutes, days, and even weeks (e.g., Cave, 1997; Cave \& Squire, 1992). These results suggest priming of long-term high-level representations, structural descriptions (Biederman, 1987; Marr \& Nishihara, 1978), perhaps, which are themselves invariant over the same transformations. In the present experiments, we sought to isolate and examine the effects of priming at an earlier level of representation, intermediate to imagebased and object- or scene-based representations (Gibson, 1950; Grossberg \& Mingolla, 1985; Kanizsa, 1979; Marr, 1982; Nakayama, He, \& Shimojo, 1995; Ramachandran $\&$ Cavanagh, 1985). The stimuli and task used to isolate this level of representation were modeled after those developed by He and Nakayama (1995) and used by McCarley and $\mathrm{He}$ (2000); they are depicted schematically and as stereograms in Figures 1 and 2. The stimuli were stereoscopic arrays of colored rectangles. The displays appeared as vertical stacks of three horizontally receding layers, oriented to recede either top away from the observer, forming a ground-like display, or bottom away, forming a ceiling-like display. The observers' task was to search the middle horizontal layer of each display for a target item distinguished by its color. These stimuli and task were useful for two reasons. First, because groundlike and ceiling-like displays differed only in a reversal of binocular disparity, changes in 3-D orientation were independent of changes in image-based features and, because stimuli were not representations of meaningful shapes or objects, they should have elicited little semantic priming. Thus, the effects of priming by 3-D layout could be isolated from the effects of priming by either twodimensional (2-D) features or by semantic properties. Second, the task to be performed demanded 3-D perceptual organization of the stimuli. Because rows of items belonging to the middle layer in each display were interlaced with rows belonging to the top and bottom layers, the items to be searched for could not be selected as a group in two dimensions. Efficient performance thus required the observers to select relevant items on the basis of their 3-D position within the stimulus array, as was confirmed by evidence that performance varies with 3-D orientation of the display relative to the observer (McCarley $\& \mathrm{He}, 2000)$.

\section{EXPERIMENT 1}

In Experiment 1, we reanalyzed previously reported data (McCarley \& He, 2000, Experiments 1 and 2) by conducting as an initial test of the possibility of priming by 3 -D stimulus organization. The observers performed a visual search task that required them to select a subset of items distributed across depth within an embedding array of distractors and to detect the presence or absence of an odd-colored item within the selected subset. In Experiment $1 \mathrm{~A}$, the rows of items to be searched were slanted in depth and were coplanar (Figure 1). In Experiment 1B, they were frontoparallel and embedded within three separate planes (Figure 2). If encoding of surface layout does facilitate subsequent encoding of a similar display, perceptual organization of stimulus displays should be achieved most easily when 3-D orientation is unchanged between trials.

\section{Method}

Observers. The observers in Experiment 1A were 5 men and 3 women, ages 19-41. The observers in Experiment 1B were 9 women and 4 men, ages 19-41. All the observers in Experiment 1A were experienced psychophysical observers. Four observers in Experiment $1 \mathrm{~B}$ had participated in Experiment 1A, whereas the rest were relatively inexperienced psychophysical observers. All the observers had normal or corrected-to-normal visual acuity and normal stereopsis. One observer in Experiment 1A and 3 in Experiment 1B were aware of the original experimental hypotheses (discussed in an earlier report, McCarley \& He, 2000). The remaining observers were naive to the original experimental hypothesis.

Apparatus and Stimuli. The stimuli were generated by a Commodore Amiga 2000, were presented at a refresh rate of $60 \mathrm{~Hz}$, and were viewed through frame-sequential haploscopic glasses. Viewing distance was approximately $80 \mathrm{~cm}$. The observers were free to move their heads.

The stimuli were modeled after those in He and Nakayama (1992, Experiment 2) and are schematically depicted in Figure 1 (Experiment 1A) and Figure 2 (Experiment 1B). Each display comprised a stereoscopically created $4 \times 3 \times 3$ array of rectangular orange and green elements, displayed against a black background. In Experiment 1A, individual elements were stereoscopically slanted and arranged to form three implicit horizontal planes, stacked vertically and receding in depth. In Experiment 1B, individual elements were frontoparallel and were arranged to form three horizontal strata, stacked vertically and receding in depth. Each horizontal array (implicit plane or stratum) comprised three rows of four elements, with the middle row of each array centered at zero disparity. The elements were arranged so that, from the top of the display, Rows 1, 2, and 4 formed a single horizontal array, Rows 3, 5 , and 7 another horizontal array, and Rows 6, 8, and 9 a third horizontal array. Thus, the rows of elements belonging to different horizontal arrays were interlaced from the top to the bottom of the display, and the three horizontal arrays in each display could only be segregated stereoscopically. Successive horizontal arrays were separated vertically by $107 \mathrm{~min}$ arc. Successive rows within a horizontal array were separated by a center-to-center vertical distance of $47 \mathrm{~min}$ arc and by a center-to-center disparity of $22 \mathrm{~min}$ arc. Within 
A.

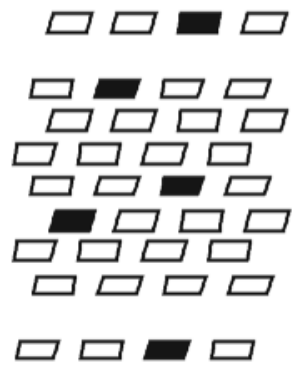

B.

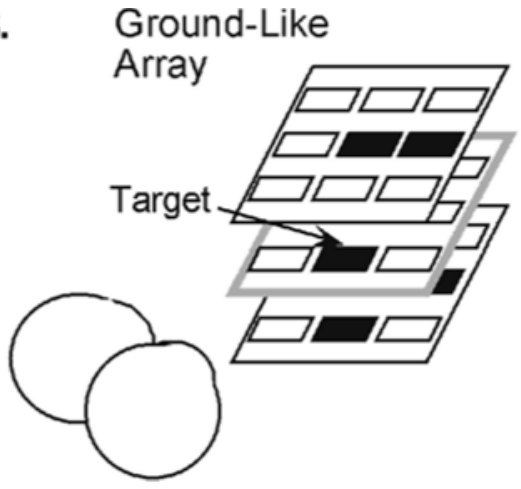

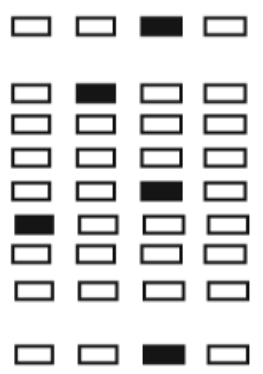
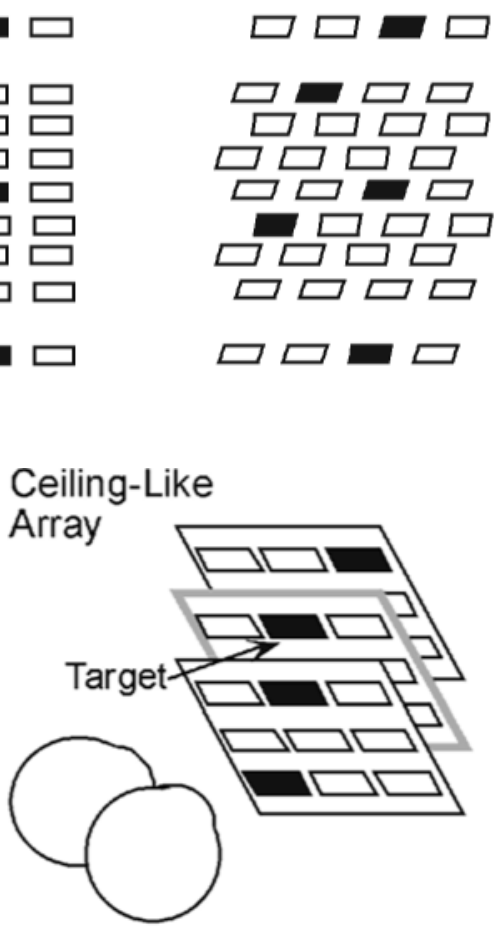

Figure 1. (A) Stereogram illustrating stimuli from Experiment 1A. Parallel fusion of left and middle arrays or cross fusion of middle and right arrays, revealing ceiling-like stimuli. Parallel fusion of middle and right arrays or cross fusion of left and middle arrays, revealing ground-like stimuli. (B) Schematic illustration of stimuli employed in Experiment 1A. Observers were asked to search the middle horizontal layer for an odd-colored item. Items within each horizontal layer of the display were coplanar.

a row, the horizontal separation between elements was varied randomly from 93 to 108 min arc. In Experiment 1A, the individual stimulus elements presented to one eye were rectangles of $54 \times$ $14 \mathrm{~min}$ arc, whereas those presented to the other eye were of approximately the same dimensions, but sheared so as to create a stereoscopic slant of $6 \mathrm{~min}$ arc from the top to the bottom of the element. For Experiment 1B, the individual stimulus elements presented to each eye were rectangles of $54 \times 14 \mathrm{~min}$ arc and thus had no stereoscopic slant. The ground-like displays were formed by orienting the horizontal arrays of elements top away in depth, so that the top row within the plane was of uncrossed disparity, the middle row was centered at disparity, and the bottom row was of crossed disparity. The ceiling-like displays were formed by orienting implicit planes bottom away in depth.

All the items within the top and bottom horizontal arrays of a display were considered as distractors, and the target of search, when present, was located within the middle horizontal array. The target was randomly assigned either the color orange or the color green. The remaining elements in the middle horizontal array were assigned the alternative color (i.e., green or orange). In both of the top and bottom arrays, there were two, four, or six elements of the same color as the target, whereas the rest were of the alternative color. The displays for target-absent trials were the same as those for target-present trials, except that the elements in the middle horizontal array were all the same color.

A fixation cross of no disparity appeared between trials, centered at the same location as the search display.

Procedure. The observers initiated each trial with a keypress. After a variable delay of $500-1,000 \mathrm{msec}$, the search display appeared. The orientation of the search display (either ground-like or ceiling-like), the number of target colored items within the display $(4,6$, or 12$)$, and the presence or absence of the target were varied randomly from trial to trial, with the constraint that all combinations of these conditions had to appear an equal number of times. The observers' task was to search the middle horizontal array of stimulus elements and indicate with a keypress the presence or absence of an element that differed in color from the others within that array. The observers were told that their reaction times (RTs) would be measured and that they should therefore respond as quickly as possible while maintaining high accuracy. After completing a short block of practice trials, each observer completed a total of 600 data-collection trials, 50 target-present and 50 targetabsent trials for each combination of stimulus orientation and number of target-colored distractors.

\section{Results}

The results of the present analysis are presented in Figure 3. RTs for incorrect responses and for trials following incorrect responses were discarded from analysis. Across observers, 3-D stimulus orientation was repeated on a mean of $45 \%$ of all trials in Experiment $1 \mathrm{~A}$ and on a mean of $46 \%(S D=2 \%)$ of all trials in Experiment $1 \mathrm{~B}$. The data for each experiment were analyzed with separate $2 \times 2 \times 2$ repeated measures analysis of variance (ANOVAs), with current trial's 3-D orientation (groundlike vs. ceiling-like), preceding trial's orientation relative to that of the current trial (same vs. reversed), and target presence (present vs. absent) as factors. As re- 
A.
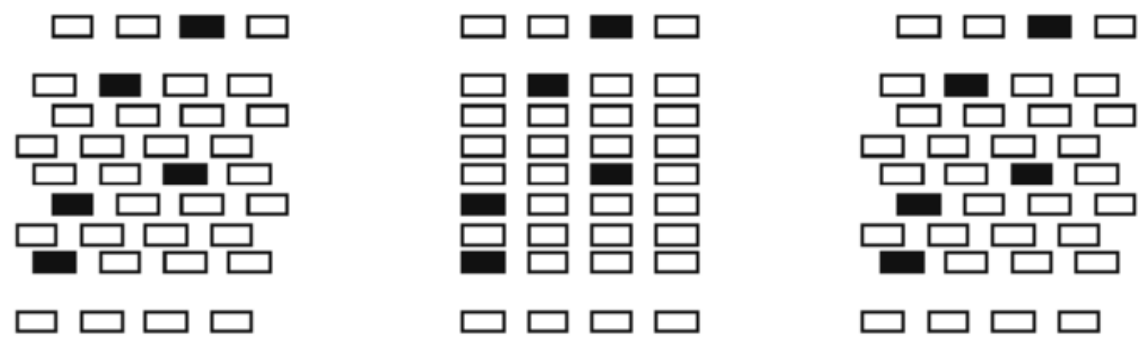

B.

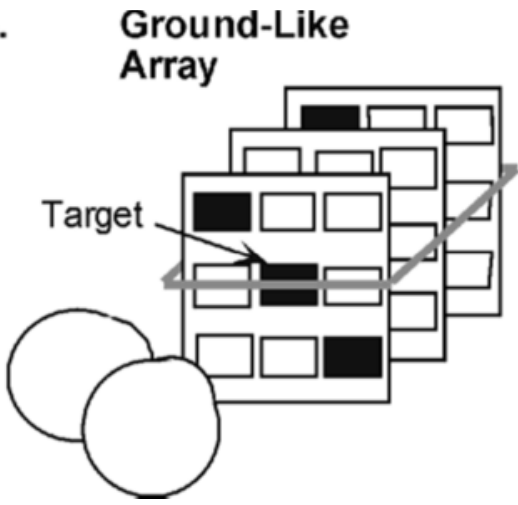

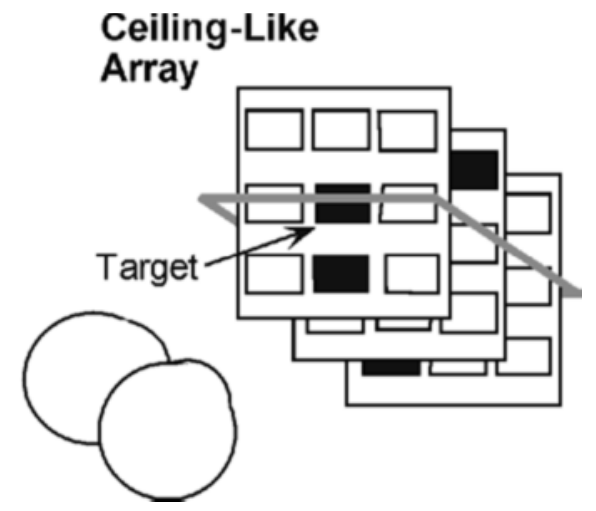

Figure 2. (A) Stereogram illustrating stimuli of Experiment 1B. (B) Schematic illustration of stimuli employed in Experiment 1B. Displays were identical to those in Experiment 1A, except that local elements were frontoparallel, and elements within a single strata were therefore not coplanar.

ported elsewhere (McCarley \& He, 2000), the RTs in both experiments were shorter for ground-like than for ceiling-like displays, and they were shorter overall for the stimuli in Experiment 1A, wherein the multiple rows to be searched were coplanar, than they were for the stimuli of Experiment 1B, wherein the rows to be searched were noncoplanar (see also He \& Nakayama, 1995). Of most importance, priming by 3-D orientation was evident in the RTs for both Experiment 1A $(M=106 \mathrm{msec})$ and Experiment 1B $(M=129 \mathrm{msec})$. Analysis of the RTs in Experiment 1A produced a reliable main effect of 3$\mathrm{D}$ orientation, indicating faster RTs for ground-like displays $[F(1,7)=12.19, p=.01]$, a reliable main effect of target presence, indicating faster RTs for target-present displays $[F(1,7)=23.17, p=.002]$, and a reliable main effect of the preceding trial's orientation, indicating faster RTs when stimulus orientation was repeated between trials $[F(1,7)=12.01, p=.01]$. Analysis of error rates from Experiment 1A produced a reliable main effect of target presence $[F(1,7)=18.36, p=.004]$, indicating more accurate responses with target-absent displays and a reliable interaction of 3-D orientation with the preceding trial's orientation $[F(1,7)=8.13, p=.025]$, reflecting a larger difference between error rates for groundlike and ceiling-like displays when stimulus orientation was changed between trials than when the same orientation was repeated. There was no indication that the ef- fects of priming by 3-D orientation were the result of a speed-accuracy trade-off. Analysis of the RTs in Experiment 1B likewise confirmed that responses were faster for ground-like stimuli than for ceiling-like stimuli $[F(1,12)=8.30, p=.014]$ faster for target-present than for target-absent trials $[F(1,12)=33.83, p<.001]$, and faster when 3-D orientation was repeated than when orientation was reversed $[F(1,12)=23.96, p<.001]$. Analysis of error rates produced a reliable main effect of target presence $[F(1,12)=26.56, p<.001]$, indicating greater accuracy in the target-absent trials and a reliable three-way interaction $[F(1,12)=5.09, p=.044]$, but again there was no evidence that a speed-accuracy tradeoff could account for the decreases in RTs that occurred with repetition of 3-D orientation.

Overall, the results indicate substantial perceptual priming by repetition of surface orientation. Importantly, the changes in stimulus orientation were not correlated with changes in response or with changes in 2-D image features (e.g., target color or vertical and horizontal target location), or, in Experiment 1B, with changes in the 3-D orientation of individual stimulus elements. Priming by global 3-D orientation therefore cannot be an artifact of priming by any of these properties. The facilitation observed thus seems most plausibly to represent priming of 3-D perceptual organization-that is, the observer's ability to determine the spatial layout and achieve 
Experiment 1a

Items Coplanar
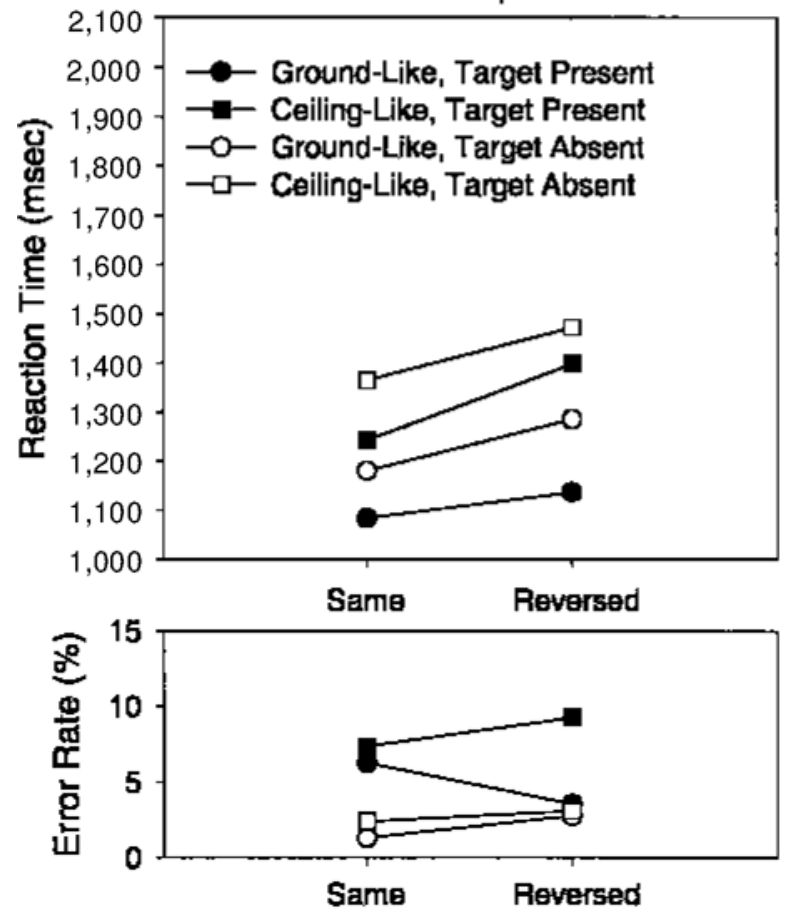

Experiment 1b Items Frontoparallel
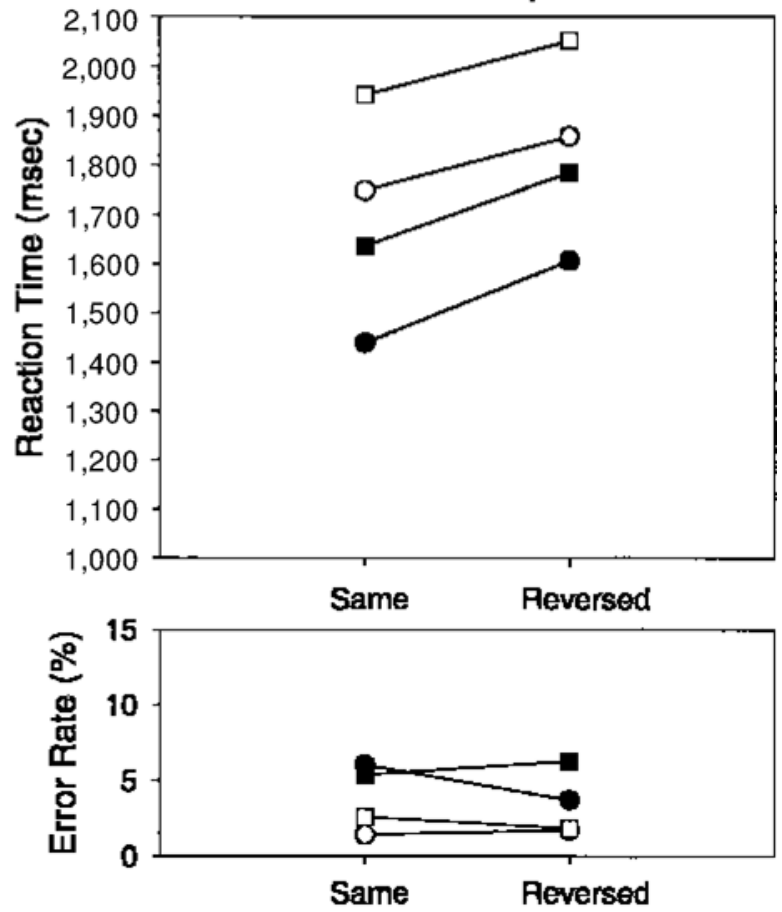

\section{3-D Orientation Relative to Preceding Trial}

Figure 3. Mean RTs and error rates for Experiments $1 \mathrm{~A}$ and $1 \mathrm{~B}$. With stimuli of either experiment, RTs were longer following a reversal of three-dimensional stimulus orientation between trials.

appropriate perceptual groupings of items within a display. In order to find more direct support for this conclusion, an additional analysis was conducted on the RTs of Experiment 1B. Here, the only RTs we considered were those for the detection of targets that were located, on a second or third consecutive trial, within the middle row of the display. Because ground-like and ceiling-like displays were both centered at zero disparity and at the same vertical position, and because individual stimulus elements within Experiment 1B were always frontoparallel, reversals of surface orientation in this experiment did not entail strictly local changes within the middle row of the search array. ${ }^{1}$ Therefore, for targets located twice consecutively within the middle row, any priming effected by repetition of 3-D orientation would clearly reflect priming by the 3-D organization of the stimulus as a whole, and not priming by any strictly local target features. The data for 1 observer, for whom the target never fell twice consecutively in the middle row without a concomitant change in display orientation, was excluded from this analysis. Data for the remaining observers were collapsed across displays of ground-like and ceiling-like orientations, and the mean RT to stimuli preceded by a display of 3-D orientation was compared with the mean RT to stimuli preceded by a display of the opposite orientation. Priming by 3-D orientation was evident. Mean facilitation under these conditions was $215 \mathrm{msec}[t(11)=$ $2.54, p=.027]$.

\section{EXPERIMENT 2}

The results of Experiment 1 demonstrate that simple repetition of orientation in depth can facilitate perceptual organization of complex 3 -D displays. Experiment 2 was conducted in order to examine this effect more closely and to begin to specify its locus. It is possible that the priming observed in Experiment 1 reflected facilitation within a low-level sensory representation, rather than within a higher-level visual surface representation. In order to test this possibility, we replicated Experiment 1B, but on some trials masked the stimuli following the observer's response. A significant reduction of sequential priming by poststimulus masking would suggest priming by purely sensory mechanisms, whereas a demonstration of trial-to-trial facilitation by repetition of 3-D orientation, despite poststimulus masking, would suggest priming by a higher-level representation. As an additional modification to the procedure in Experiment 1B, the stimuli in Experiment 2 were not constrained in such a way that all levels of 3-D orientation, target presence or 
absence, and number of target-colored distractors were required to appear equal numbers of times. Instead, these properties were chosen randomly for each trial. This eliminated any contingencies between the stimuli that appeared from trial to trial and allowed the effects of priming across longer sequences of trials to be examined.

\section{Method}

Observers. The observers were 9 graduate and undergraduate students, ages 21-35 years. All the observers had normal or correctedto-normal visual acuity and normal stereopsis. All observers were naive to the purpose of the study.

Stimuli. The stimuli were identical to those in Experiment 1B, except for the following change. Within alternating blocks of trials, the search displays were overwritten by a poststimulus mask that immediately followed the observer's response. The mask for a given trial was identical to the stimulus array that had been presented in that trial, except that each row of elements was randomly shifted to a depth of 22 min arc crossed disparity, 0 min arc disparity, or 22 min arc uncrossed disparity. Thus, the mask for a given trial differed only in 3-D structure, and not in image-based features, from the search display of the same trial. Within blocks of unmasked trials, the search displays were replaced by a fixation cross immediately following the observer's response.

Procedure. The procedure was similar to that in Experiment 1, except for the following changes. In order to provide greater control over the amount of time separating priming from primed trials, Experiment 2 was force-paced, with trials separated by a randomly chosen response-stimulus (RS) interval of 2,000-2,500 msec. In masked trials, a poststimulus mask appeared immediately following the observer's response. The mask remained visible for an interval of $1,000 \mathrm{msec}$ and was then replaced by a fixation cross that remained visible until the onset of the succeeding trial's search display. In unmasked trials, the fixation cross appeared immediately following the observer's response and remained visible until onset of the succeeding trial's display. For all trials, a warning tone was presented 500-1,000 msec preceding the onset of the search display. The orientation of the stimulus display, the presence or absence of a target, the target's color, and the number of target-colored distractors $(2,4$, or 6$)$ within the top and bottom strata were all chosen randomly for each trial.

The observers' task was to search the middle horizontal stratum of the stimulus display for an odd-colored element and to indicate the target's presence or absence with a keypress. The observers were told that their RTs would be recorded and that they should therefore respond as quickly as possible while maintaining accuracy. Auditory feedback was presented following incorrect responses. After performing a short block of warm-up trials, each observer completed six blocks of 100 trials with alternating blocks of masked and unmasked trials. The order of masked and unmasked blocks was counterbalanced across observers.

\section{Results}

The mean RTs and error rates are presented in Figure 4. The RTs for incorrect responses and for the trials following incorrect responses were discarded. All other RTs were subjected to a $2 \times 2 \times 2 \times 2$ repeated measures ANOVA, with current trial's 3-D orientation (ground-like vs. ceiling-like), preceding trial's orientation relative to that of the current trial (same vs. reversed), masking (masked vs. unmasked), and target presence (present vs. absent) as factors. Overall, the RTs were shorter for ground-like than for ceiling-like displays (mean advantage $=136 \mathrm{msec})[F(1,8)=9.56, p=.015]$, shorter for target-present than for target-absent responses $[F(1,8)=32.69, p<.001]$, and, confirming the results of the previous experiment, were shorter when 3-D orientation was repeated between trials (mean advantage $=$ $116 \mathrm{msec})[F(1,8)=26.31, p=.001]$. Analysis failed, however, to reveal a reliable main effect of poststimulus masking $[F(1,8)=1.98, p=.197]$, suggesting that RTs were not generally longer in the masked than in the unmasked blocks of trials. Most important, the data gave little indication that poststimulus masking reliably interacted with orientation of the preceding trial's display $(F<1)$, demonstrating that a passively viewed, taskirrelevant masking stimulus interposed between displays did little to attenuate trial-to-trial priming by $3-\mathrm{D}$ organization. This suggests that priming by trial-to-trial stimulus repetition did not arise from a purely sensory or iconic memory trace (which should have been degraded by the presentation of the poststimulus display) but rather from a higher-level representation immune to interference from a task-irrelevant sensory mask. No additional effects found in the RT data were statistically reliable. An analysis of the error rate data indicated only one reliable effect-a main effect of response, confirming that the observers made fewer errors when no target was present $[F(1,8)=16.13, p=.004]$. There was no evidence that a speed-accuracy trade-off could have accounted for the priming evident in the RT data.

Overall, the results indicate that priming by repetition of surface orientation was largely immune to interference from the poststimulus masks employed here. The facilitation enacted by viewing and responding to a stimulus, that is, endured through the subsequent presentation of a passively viewed stimulus, as predicted by the hypothesis that priming results from the persistence of attentional weights and not from the persistence of a sensory representation itself. The same hypothesis, however, suggests that the effects of priming should not endure for extended lengths of time, as might result from long-term retention of a representation of a particular stimulus (see, e.g., Chun \& Jiang, 1998; Lassaline \& Logan, 1993), but should diminish over short periods of time as additional stimuli are actively encoded. For an estimate of the full duration of priming by a single stimulus, the target-present data were subjected to the memory kernel analysis procedure (Maljkovic \& Nakayama, 1994, 1996). Figure 5 presents the mean amount of priming measured for all targetpresent trials, as a function of the priming trial's temporal relation to the current trial. The values on the abscissa indicate the position of the priming trial relative to the present trial: position -1 indicates the immediately preceding trial, position -2 represents the penultimate trial preceding the current trial, and so forth. The values on the ordinate indicate the amount of priming enacted by the trial in question, collapsed across all sequences of in- 

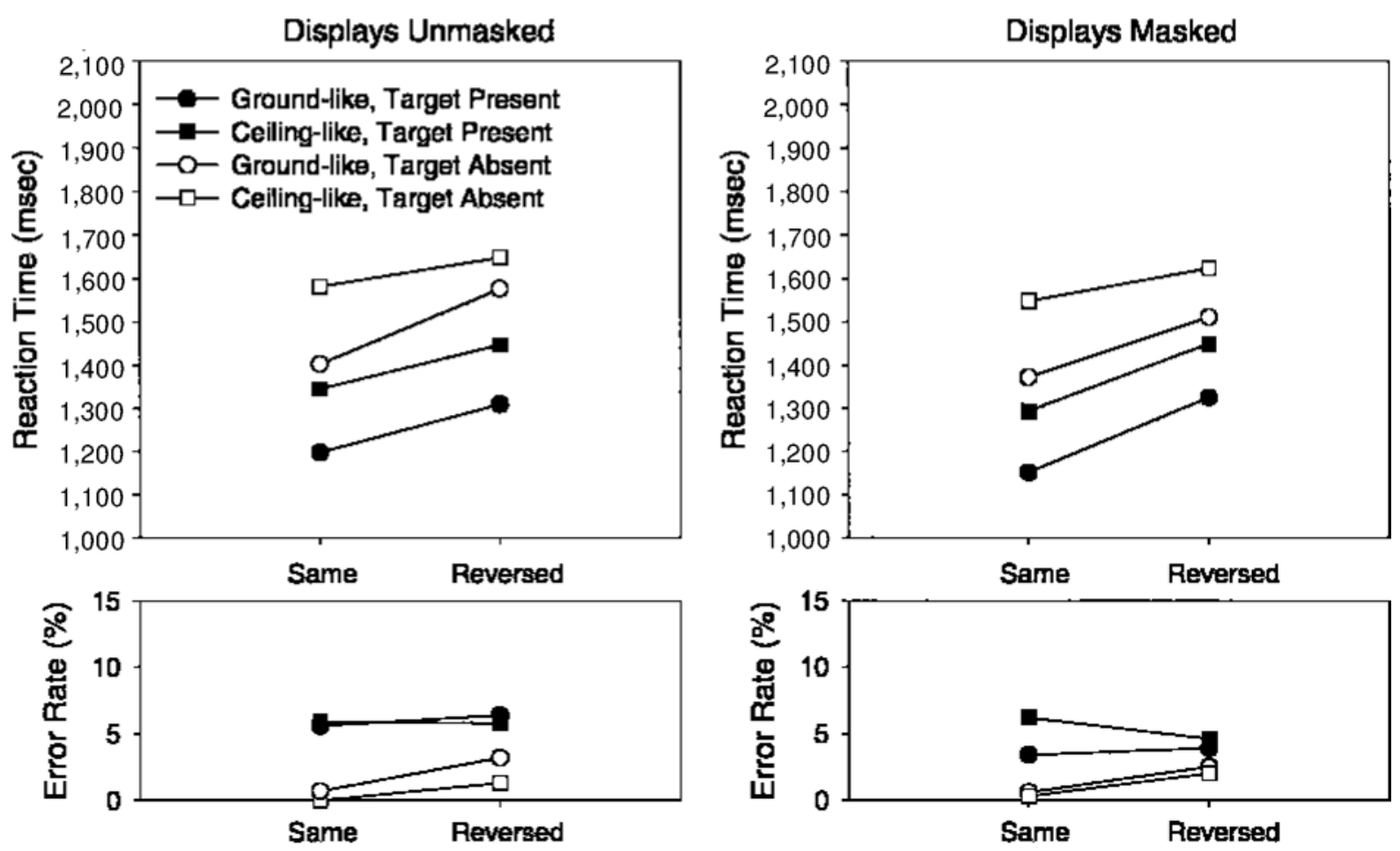

\section{3-D Orientation Relative to Preceding Trial}

Figure 4. Mean reaction times and error rates for unmasked and poststimulus masked stimuli of Experiment 2. Priming by trialto-trial repetition of three-dimensional (3-D) orientation was not reduced by the presentation of a passively viewed 3-D poststimulus mask.

terposed trials. The Y-value for position -3 , for example, indicates the mean RT of all trials for which the third preceding trial was of the opposite orientation minus the mean RT of all trials for which the third preceding trial was of the same orientation, independent of the intervening trials' orientations. A positive value for this data point indicates that the RTs on a given trial were faster when the third preceding trial was of the same orientation as the current trial. The data indicate that the priming affected by a single stimulus could persist beyond the immediately succeeding trial; for at least 2-3 trials following the presentation of a stimulus, the RTs for target detection in another display of the same orientation were shortened. Furthermore, the persistence of priming was similar for masked and unmasked displays. These results reaffirm that priming was not the result of a persisting sensory representation, which should have been obligatorily destroyed by either incidental or attentive encoding of a subsequent stimulus. The priming affected by a single stimulus, however, did fall to negligible levels within approximately four trials. This fairly rapid decay serves to distinguish the phenomena examined here from the effects of priming by stored, long-term representations; this accords well with the proposition that priming is enacted through short-term manipulation of attentional weights assigned to stimulus properties.

\section{EXPERIMENT 3}

In Experiments 1 and 2, it was demonstrated that attentional encoding of a 3-D surface layout can prime subsequent encoding of stimuli in the 3-D orientation. It is not clear, however, what details of the stimulus, aside from 3-D orientation, might affect this priming. Maljkovic and Nakayama (1996) found that priming by target features (i.e., color and spatial frequency) and target location were additive in a visual search task. Similarly, Robertson (1996) found priming by the attended level (global vs. local) in hierarchical displays (Navon, 1977), independent of stimulus location. Robertson also found, however, that variation of attributes other than location could sometimes mediate the effects of priming by hierarchical level, even if the manipulated attributes were task irrelevant and were varied independently of attended level; more specifically, irrelevant changes in stimulus polarity, contrast, or color, reduced the priming of attention to the global level in global/local displays. It is therefore unclear 


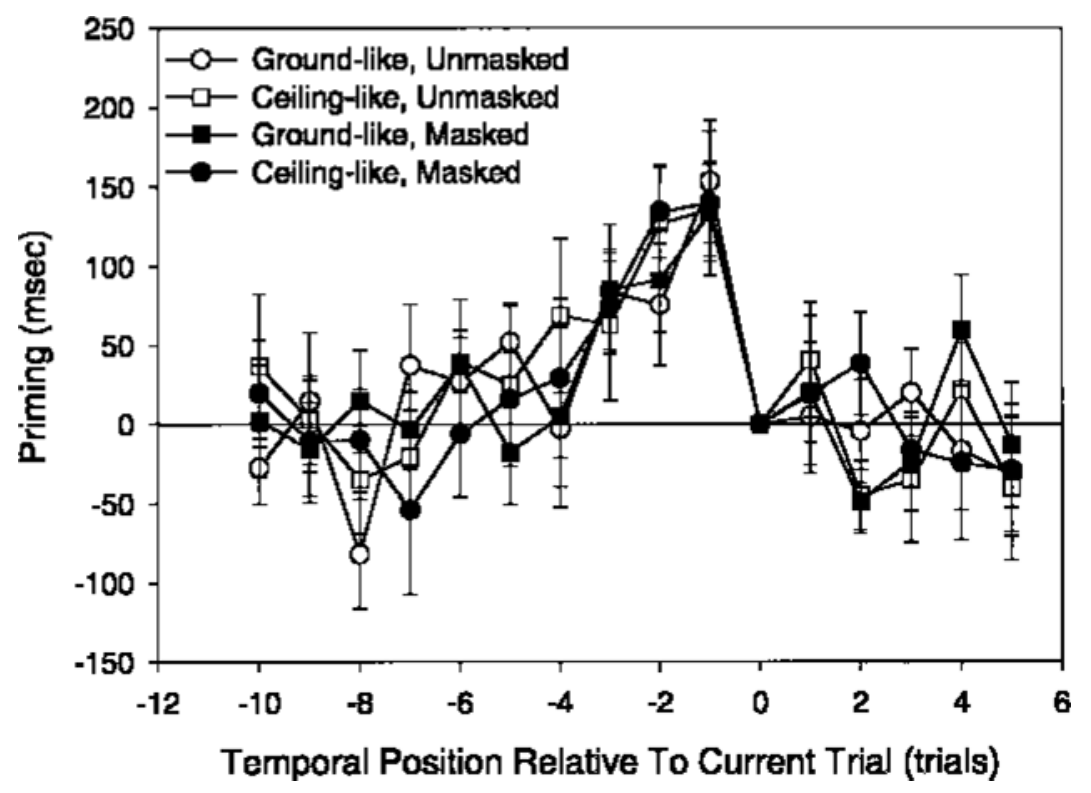

Figure 5. Memory kernel analysis (Maljkovic \& Nakayama, 1994) of data from Experiment 2 . The graph indicates the magnitude of priming affected by a stimulus as a function of the number of trials separating primed stimulus from priming stimulus. Position -1 on the abscissa indicates the trial immediately preceding the primed trial, -2 indicates the penultimate trial preceding the primed trial, and so forth. Positions 1-5 indicate the amount of "priming" affected by trials subsequent to the primed trial and are included to provide a measure of baseline variability in priming. Error bars indicate $\pm 1 S E$.

how variation of orthogonal stimulus properties should be expected to affect the benefits of priming by 3-D orientation with the present stimuli. The results of Experiments 1 and 2 indicate that priming by 3-D orientation is not wholly predicated on the repetition of additional stimulus attributes; given that target color and the layout of colored distractors changed randomly from trial to trial, independently of changes in stimulus orientation, priming by surface layout clearly did not hinge on the concomitant repetition of target color. Nonetheless, it is possible that variation of such stimulus properties might have moderated the facilitation obtained from the repetition of surface layout. In Experiment 3, we tested this possibility by examining the concomitant effects of repeated target color and 3-D orientation.

In order to measure the effects of priming by target color, we modified the experimental paradigm of the preceding experiments in two ways. First, we adapted the visual search task employed by Bravo and Nakayama (1992) and Maljkovic and Nakayama $(1994,1996)$ for use with stimulus displays like those in the preceding experiments. Rather than requiring the observers to determine whether a target was present or absent, this task required the observers to make a judgment of the target's shape. This allowed a target to be presented in every trial and thus prevented target-absent trials from diluting any effects of priming by target color. Second, to disentangle effects of priming by target color from the potential effects of priming by distractor color, we presented only gray distractors within the middle stratum of the display and randomly assigned the colors orange and green to individual distractors within the top and bottom strata.

\section{Method}

Observers. The observers were 11 undergraduate students, ages 18-31 years. All the observers had normal or corrected-to-normal visual acuity and normal stereopsis. All were naive to the purpose of the study.

Stimuli. The stimuli were similar to those in Experiment 1B, except for the following changes. Nontarget elements within the middle horizontal stratum of the search displays were no longer of the alternative target color (i.e., orange or green), but were always gray. The target element itself was not rectangular, but had a small region clipped from the top and bottom corners of one side to form an arrow that pointed either leftward or rightward. As in the previous experiment, the individual distractors within the top and bottom horizontal arrays of the search displays could be of either target color (i.e., orange or green). In order to avoid possible effects of correlation between the color of the target element and the density of targetcolored elements within the top and bottom arrays of the stimulus display, the color of each element within the top and bottom arrays was randomly chosen. Each element was equally likely to be of either color. All stimulus displays in Experiment 3 were unmasked.

Procedure. The experimental task was patterned after that of Bravo and Nakayama (1992) and Maljkovic and Nakayama (1994, 1996). The observers' task was to indicate in each trial whether the colored element within the middle horizontal stratum of the display 


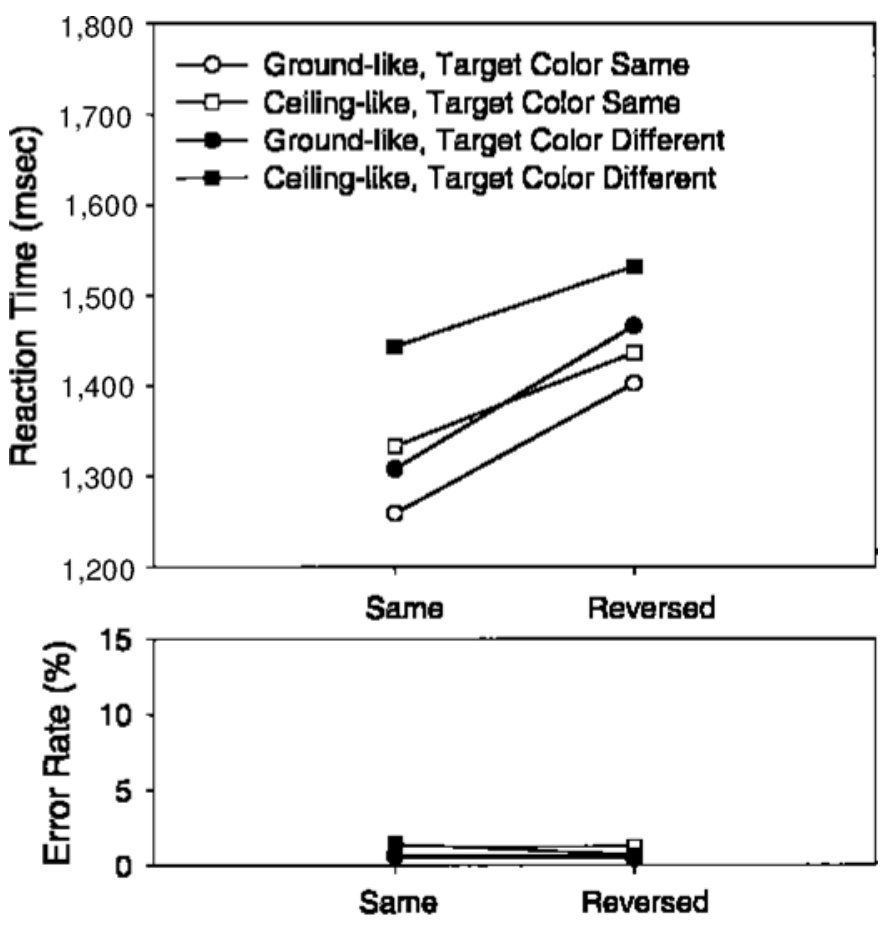

\section{3-D Orientation Relative to Preceding Trial}

Figure 6. Mean reaction times (RTs) and error rates for Experiment 3. RTs were reduced by trial-to-trial repetition of either three-dimensional (3-D) stimulus orientation or target color. However, priming by 3-D orientation and by target color were independent.

pointed leftward or rightward. A target was present in each trial, and the direction in which the target pointed (leftward or rightward) was randomly chosen. The observers were informed that their RTs would be measured and that they should respond as quickly as possible while maintaining accuracy. Auditory feedback was provided after incorrect responses. The trials were separated by an RS interval of $1,000 \mathrm{msec}$, and a warning tone was presented $500 \mathrm{msec}$ prior to the onset of each display. After completing a short block of warm-up trials, each observer completed 10 experimental blocks of 50 trials each.

\section{Results and Discussion}

Mean RTs and error rates are presented in Figure 6. The RTs for incorrect responses and for trials following incorrect responses were discarded. All other RTs and error rates were subjected to $2 \times 2 \times 2$ repeated measures ANOVAs, with current trial's 3-D orientation (ground like vs. ceiling like), preceding trial's orientation relative to that of the current trial (same vs. reversed), and preceding trial's target color relative to that of the current trial (same vs. different) as factors. Once more, RTs were reliably shorter for ground-like than for ceiling-like displays (mean advantage $=84 \mathrm{msec})[F(1,10)=12.16, p=.006]$ and when 3-D orientation was repeated between trials $($ mean advantage $=126 \mathrm{msec})[F(1,10)=20.83, p=$
.001]. Furthermore, consistent with the report of Maljkovic and Nakayama (1994), RTs were reduced significantly when target color was repeated between trials $($ mean advantage $=82 \mathrm{msec})[F(1,10)=18.82, p=.001]$. The effects of priming by surface orientation and target color, however, were apparently additive, as the interaction between the preceding trial's orientation and target color failed to approach significance $(F<1)$. The RT data indicated that no additional interactions reached significance. Statistical analysis of the error rates failed to reveal any reliable effects, giving no evidence that the priming evident in the RT data could have resulted from a speedaccuracy trade-off.

In sum, we found in Experiment 3 facilitation of performance for both trial-to-trial repetition of 3-D stimulus orientation and trial-to-trial repetition of target color. Priming by repetition of 3-D orientation and by target color, however, were independent. This suggests that the facilitation of 3-D perceptual organization was achieved by priming of the 3-D layout itself, and not by priming of a more general, multifeatured stimulus representation. The present evidence is thus in accord with previous demonstrations of independent priming by multiple stimulus characteristics. 


\section{EXPERIMENT 4}

Experiment 4 was conducted to measure the strength of volitional control that observers might exert over priming by $3-\mathrm{D}$ stimulus organization. In each of the previous experiments, 3 -D orientation of the stimulus displays was varied randomly and without warning. The observers therefore could not anticipate or prepare for a display of either orientation prior to stimulus presentation. It is thus possible that priming was obtained only because the observers, absent motivation to do otherwise, voluntarily retained attentional settings trial to trial. Past research, however, has suggested that priming similar to that observed here occurs automatically and obligatorily. Lamb and colleagues, for example, found that repetition of the to-be-attended level facilitated RTs for hierarchical stimuli even when the relevant level was predictable trial to trial (Lamb et al., 1998) or was validly cued prior to stimulus onset (Lamb et al., 2000). Similarly, Maljkovic and Nakayama (1994) found that predictability of the target-defining feature in visual search could not overcome the effects of trial-to-trial priming by feature values. To measure the strength of volitional control that observers might effect over the priming observed here, we replicated Experiment 3, except that an auditory cue was presented prior to each trial to signal the 3-D orientation of the impending display. If priming by $3-\mathrm{D}$ layout is at all volitional, the effects of repeated orientation should be reduced. In contrast, if priming of surface layout is wholly automatic, priming should be of roughly the same magnitude as that observed in Experiment 3.

\section{Method}

Observers. The observers were 11 undergraduate students, ages 17-22 years. All the observers had normal or corrected-to-normal visual acuity and normal stereopsis. All were naive to the purpose of the study.

Stimuli and Procedure. The stimuli and procedure were identical to those in Experiment 3, except that the alerting tone preceding each trial was replaced by a tone with a frequency of either $750 \mathrm{~Hz}$ or $800 \mathrm{~Hz}$, which indicated, with $100 \%$ validity, the orientation of the upcoming display. The observers were informed that the tone preceding each trial would indicate the orientation of the upcoming display and that they should use the tone to anticipate and prepare for a display of the appropriate orientation.

\section{Results and Discussion}

Mean RTs and error rates are presented in Figure 7. RTs for incorrect responses and for trials following incorrect responses were discarded. RTs and error rates were submitted to $2 \times 2 \times 2$ repeated measures ANOVAs, with current trial's orientation, preceding trial's orientation relative to that of the current trial, and preceding trial's target color relative to that of the current trial, as factors. The RTs for the ground-like stimuli were again shorter than those for the ceiling-like stimuli (mean advantage $=42 \mathrm{msec})[F(1,10)=5.06, p=.048]$, and repetition of target color again reduced search times (mean advantage $=67 \mathrm{msec})[F(1,10)=14.07, p=.004]$. All in- teractions fell short of significance. However, of foremost importance, the RTs in Experiment 4 were again shorter following repetition of 3-D orientation (mean advantage $=121 \mathrm{msec})[F(1,10)=22.34, p=.001]$. Analysis of error rates revealed no reliable effects and provided no evidence that a speed-accuracy trade-off could have accounted for the effects observed in the RT data. The data thus demonstrate that foreknowledge of surface orientation could not overcome the effects of priming by the preceding display. This conclusion is corroborated by a direct comparison of the magnitudes of priming observed in both Experiments 3 and 4. For this analysis, the data from the last two experiments were submitted to a mixed ANOVA, with level of cuing (3-D orientation cued vs. uncued, i.e., Experiment 3 vs. Experiment 4) as the between-subjects factor, and with current trial's orientation, preceding trial's orientation relative to that of the current trial, and preceding trial's target color relative to that of the current trial as the within-subjects factors. As was expected, the results indicated reliable priming by both 3-D orientation and target color, as well as a significant benefit of ground-like displays (all $p \mathrm{~s}<.002$ ). The results also indicated a reliable three-way interaction of current trial's orientation $\times$ preceding trial's orientation $\times$ level of cuing $[F(1,20)=4.79, p=.041]$, reflecting the fact that priming was greater for the ground-like than for the ceiling-like displays in Experiment 3, but greater for the ceiling-like than for the ground-like displays in Experiment 4 . There was little indication, however, that foreknowledge of stimulus orientation reduced mean RTs $(F<1)$ and, accordingly, there was no indication that cuing of 3-D orientation reduced the magnitude of priming by 3 -D layout $(F<1)$. As can be seen in Figure 8, the strength of priming by $3-\mathrm{D}$ orientation differed very little between the last two experiments.

In full, the data suggest that neither the ease of perceptually organizing the present stimuli nor the effects of priming by 3-D stimulus layout was strongly modulated by the observers' foreknowledge of display orientation and indicate that the priming observed here was not mediated by volitional processes.

\section{GENERAL DISCUSSION}

Using a visual search task that required observers to perceptually segregate and select a subset of items from within an embedding of a 3-D array of distractors, in the present series of experiments, we found that perceptual organization of complex 3-D displays could be facilitated by trial-to-trial repetition of display orientation. When displays, oriented either ground like or ceiling like relative to the observer, reversed orientation between trials, RTs were reliably longer than when displays presented on successive trials were of the same orientation. Several factors collude to specify the nature of this priming. Differences in 3-D orientation were created with simple reversals of binocular disparity, so that ground-like and 


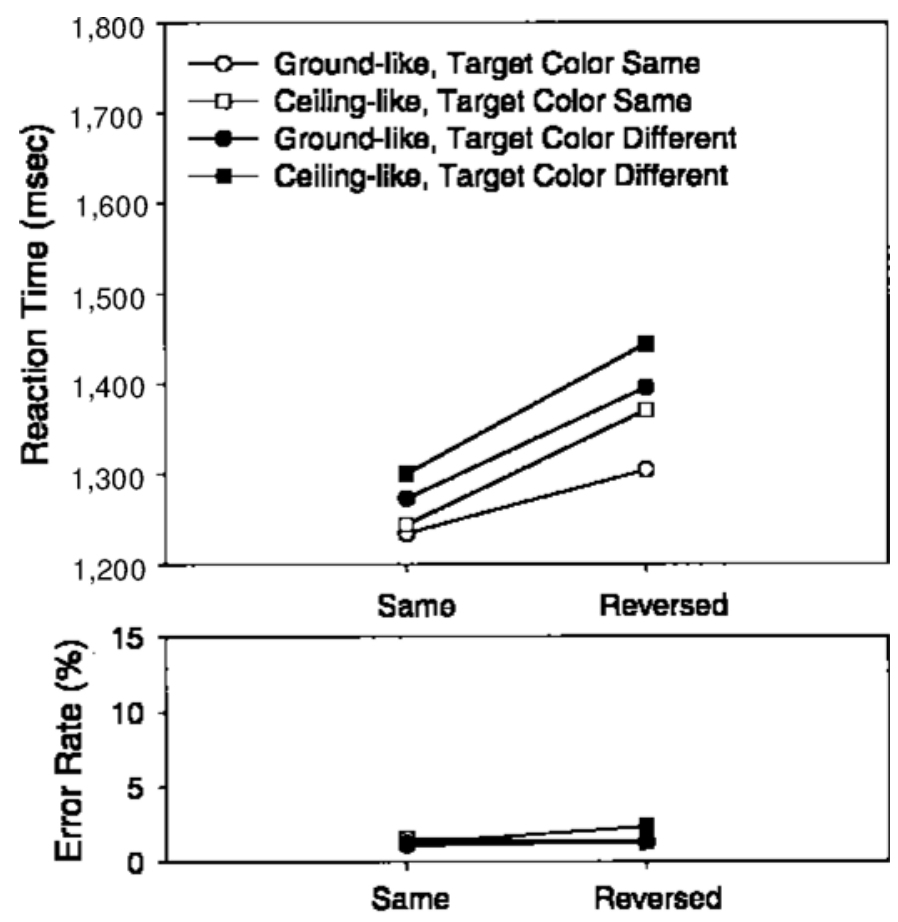

\section{3-D Orientation Relative to Preceding Trial}

Figure 7. Mean reaction times (RTs) and error rates for Experiment 4. Priming by repetition of three-dimensional (3-D) stimulus orientation was evident, despite the fact that observers were provided foreknowledge of each display's 3-D orientation.

ceiling-like displays did not differ consistently in any purely image-based features. The facilitation that was produced by the repetition of surface orientation must therefore have been rooted at, or beyond, the level of representation within which 3-D stimulus layout is derived from the integration of 2-D image features. This conclusion is reinforced by the observation that reversal of 3-D orientation increased RTs even when the target itself appeared twice consecutively at zero disparity and at the same height within the display. The results also suggest that the priming by surface layout was not produced by a purely sensory or iconic representation; facilitation by stimulus repetition was apparent even when 3-D poststimulus masks were interposed between trials and when multiple trials intervened between the priming and the primed stimulus. Finally, the data indicate that priming by 3-D layout occurred independent of priming by target color and that it was not dependent on the observers' knowledge or expectations of an upcoming display's orientation.

Other such perceptual effects, of course, are already familiar. Recently, for example, Sanocki and Epstein (1997) asked observers to judge the spatial relationship between two objects in a scene and found that responses were faster when the observers could briefly preview the scene absent the target items. As noted, a number of researchers have found that observers are faster in responding to hierarchical stimuli (Navon, 1977) when the level (global or local) to be attended remains constant between trials (Kim et al., 1999; Roberston, 1996; Robertson et al., 1993; Ward, 1982). Maljkovic and Nakayama (1994, 1996), as described earlier, demonstrated that trial-totrial repetition of the location or the defining feature of a target allowed observers to focally attend the target more quickly in a visual search. Atchley, Andersen, and Wuestefeld (1998) recently found that priming could facilitate the recovery of shear necessary for perception of a surface from optic flow.

The present results may be related to Robertson's (1996) hypothesis of an attentional print that persists following effortful (attentive) encoding of a visual stimulus. Robertson described the print as a trace of the attentional weights assigned to various stimulus features, which occurs while a display is parsed into groups, objects, or channels for higher-level selection. By maintaining the weights necessary for perceptual organization of a given display, the trace would allow a similar, succeeding display to be organized with greater efficiency. For example, an attentional print of the weights assigned to the low- and highspatial frequency bands might contribute to priming of 


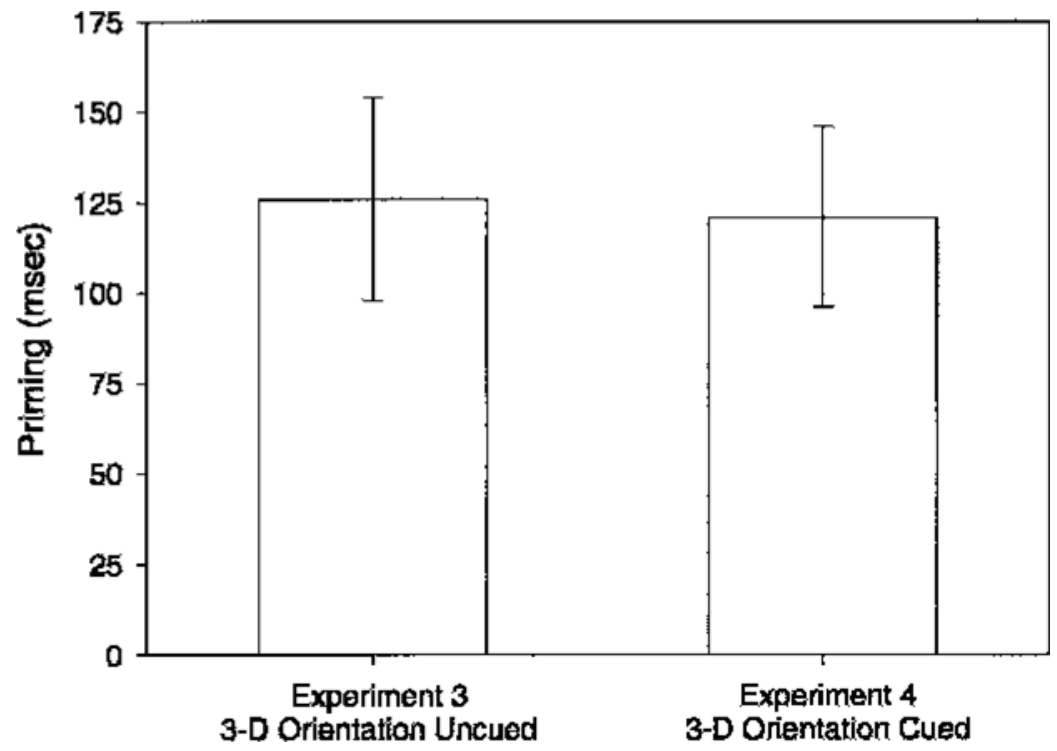

Figure 8. Mean magnitude of priming by three-dimensional (3-D) layout observed in Experiment 3, in which observers were provided no foreknowledge of stimulus orientation, and that in Experiment 4, in which observers were provided auditory cues to indicate the orientation of each impending stimulus. Values are collapsed across trials of ceiling-like and ground-like orientation and across trials in which target color was repeated or changed. Error bars indicate $\pm 1 S E$. Foreknowledge of 3-D stimulus orientation did not reduce priming by repetition of orientation.

selection by levels in hierarchical stimuli. Similarly, a print of the weights assigned to the color or spatialfrequency channels that are used to distinguish a target from distractors, or a print of the weights assigned to information coming from the target and distractor locations, could effect priming across trials in a visual search task (Maljkovic \& Nakayama, 1994, 1996). Furthermore, because the attentional print would mediate encoding of stimulus features, it would allow priming invariant over changes in the location and identity of stimulus items. To the extent that stimulus characteristics are encoded within separate neural channels, it would also allow priming of one attribute independently of another. Because the print would reflect memory for the attentional weights assigned to stimulus attributes, and would not reflect a persisting sensory representation of the attributes themselves, it would be resistant to purely sensory interference. Finally, because the attentional weights assigned to stimulus properties would be reassigned as necessary to encode new input, the priming affected by a single stimulus would diminish as new stimuli are attentively encoded.

As described in detail above, the priming demonstrated in the present experiments exhibits many of the characteristics predicted by the hypothesis of an attentional print: Priming by 3-D layout was independent of priming by target color, was immune to sensory interference, and decayed as new stimuli were encoded over the course of 2-3 experimental trials. However, the present data sug- gest that an attentional print may exist for stimulus characteristics that are more abstract than simple feature values. In the experiments described here, observers were asked to parse a stereoscopic display of multiple elements into a set of three strata receding in depth from the observer's station point. This suggests that an attentional print that primed performance across repetition of 3-D stimulus orientation worked by facilitating the observers' ability to segregate and group multiple items into strata to be attended or ignored. However, the displays in the opposite 3-D orientations were distinguished only by the vertical positions of items at near or far disparities. That is, ground-like and ceiling-like displays all contained stimuli within the same range of binocular disparities and differed only in the order in which the rows of crossed and uncrossed disparity appeared from the top to the bottom of the display. Thus, trial-to-trial priming of 3-D orientation could not have been affected by priming of attention to a particular range of binocular disparities. This implies that an attentional print encodes not only coarse feature values, but more abstract stimulus characteristics, such as the spatial relations between stimuli as well.

An alternative explanation for the present results is that the trial-to-trial facilitation by 3-D orientation reflects not priming of 3-D spatial relationships between stimuli, but merely priming of highly local mechanisms selective for particular values of binocular disparity. At least two considerations, however, argue against this possibility. First, the priming described here was unaffected by poststimu- 
lus masking, as priming by early and local sensory mechanisms should have been. Second, evidence from 2-D search tasks and from studies of scene perception has also suggested that stimulus repetition can prime perception of spatial relations between stimulus elements. Specifically, Maljkovic and Nakayama (1996) asked observers to locate and focally attend an odd-colored target within a horizontal group of three items. Trial to trial, the target could appear at the same or at different positions (left, middle, or right) within the group of three items. Furthermore, the entire group of items could appear at the same or different retinal locations. Notably, priming by target location was determined largely by the target's position within the group of three stimulus items and not by the target's retinal coordinates. Using more naturalistic stimuli, Sanocki and Epstein (1997) found similar results. Observers were asked to judge the relative positions of two objects within a variety of naturalistic scenes. Prior to the onset of each imperative stimulus, observers saw either an image of the impending scene absent the critical objects or a neutral stimulus, which provided no information about the layout of the impending scene. The results indicated that previewing of the imperative scenes facilitated the observers' judgments of the spatial relations between the critical objects, despite the fact that the critical objects were not present within the priming stimuli. This was true even when the priming and imperative scenes were seen from different vantage points.

Functionally, priming that is effected by an attentional print may serve to abet adaptive, yet computationally expensive, visual processes (Chun \& Nakayama, 2000). It has often been posited that to derive a rapid and accurate representation of the distal stimulus, the visual system must invoke top-down mechanisms. These may operate, for example, to allow stored knowledge of objects' shapes to guide figure-ground segregation (Peterson, 1994) and recovery of 3-D shape (Moore \& Cavanagh, 1998), or they may guide attention toward behaviorally relevant information within a familiar scene (Chun \& Jiang, 1998, 1999). Alternatively, these mechanisms may be manifest as heuristic assumptions about the nature of the visual world that, though not infallible, are likely to be correct and can therefore provide computational shortcuts to facilitate veridical reconstruction of distal stimulus properties (e.g., Aks \& Enns, 1992; Enns \& Rensink, 1991; Ramachandran, 1988). Clearly, such processes exploit long-term regularities in the visual environment. But, just as the global visual environment is characterized by recurring objects and pervasive order, so an observer's immediate visual environment is characterized by more local regularities; that is, the identities and arrangement of objects that immediately surround a stationary observer are unlikely to change dramatically or capriciously over short periods of time. Knowledge of these short-term regularities, like knowledge of the visual environment's more enduring characteristics, can therefore be exploited in order to facilitate perceptual organization and visually guided adaptive behavior.

\section{REFERENCES}

Aks, D. J., \& ENNS, J. T. (1992). Visual search for direction of shading is influenced by apparent depth. Perception \& Psychophysics, 52, 6374.

Atchley, P., Andersen, G. J., \& Wuestefeld, A. P. (1998). Cooperativity, priming, and 3-D surface detection from optic flow. Perception \& Psychophysics, 60, 981-992.

AtTnEaVe, F. (1954). Some informational aspects of visual perception. Psychological Review, 61, 183-193.

BARLOW, H. B. (1961). Possible principles underlying the transformation of sensory messages. In W. A. Rosenblith (Ed.), Sensory communication (pp. 217-234). Cambridge, MA: MIT Press.

BARTRAM, D. J. (1974). The role of visual and semantic codes in object naming. Cognitive Psychology, 6, 325-356.

BIEDERMAN, I. (1987). Recognition-by-components: A theory of human image understanding. Psychological Review, 94, 115-147.

Biederman, I., \& CoOper, E. E. (1991). Evidence for complete translational and reflectional invariance in visual object priming. Perception, 20, 585-593.

Biederman, I., \& Cooper, E. E. (1992). Size invariance in visual object priming. Journal of Experimental Psychology: Human Perception \& Performance, 18, 121-133.

Biederman, I., \& Gehardstein, P. C. (1993). Recognizing depthrotated objects: Evidence and conditions for three-dimensional viewpoint invariance. Journal of Experimental Psychology: Human Perception \& Performance, 19, 1162-1182.

Bravo, M. J., \& NaKaYAMA, K. (1992). The role of attention in different visual-search tasks. Perception \& Psychophysics, 51, 465-472.

CAve, C. B. (1997). Very long-lasting priming in picture naming. Psychological Science, $\mathbf{8}, 322-325$.

Cave, C. B., Bost, P. R., \& Совв, R. E. (1996). Effects of color and pattern on implicit and explicit picture memory. Journal of Experimental Psychology: Learning, Memory, \& Cognition, 22, 639-653.

CAve, C. B., \& SQuire, L. R. (1992). Intact and long-lasting repetition priming in amnesia. Journal of Experimental Psychology: Learning, Memory, \& Cognition, 18, 509-520.

Chun, M. M., \& JiAng, Y. (1998). Contextual cueing: Implicit learning and memory of visual context guides spatial attention. Cognitive Psychology, 36, 28-71.

Chun, M. M., \& JiAng, Y. (1999). Top-down attentional guidance based on implicit learning of visual covariation. Psychological Science, 10, 360-365.

Chun, M. M., \& Nakayama, K. (2000). On the functional role of implicit visual memory for the adaptive deployment of attention across scenes. Visual Cognition, 7, 65-81.

Enns, J. T., \& Rensink, R. A. (1991). Preattentive recovery of threedimensional orientation from line drawings. Psychological Review, 98, 335-351.

Gibson, J. J. (1950). The perception of the visual world. Boston: Houghton Mifflin.

Grossberg, S., \& Mingolla, E. (1985). Neural dynamics of form perception: Boundary completion, illusory figures, and neon color spreading. Psychological Review, 92, 173-211.

He, Z J., \& NAKAY AMA, K. (1995). Visual attention to surfaces in threedimensional space. Proceedings of the National Academy of Sciences, 92, 11155-11159.

KANIZSA, G. (1979). Organization in vision: Essays on gestalt perception. New York: Praeger.

Kim, N., Ivry, R. B., \& Robertson, L. C. (1999). Sequential priming in hierarchically organized figures: Effects of target level and target resolution. Journal of Experimental Psychology: Human Perception \& Performance, 25, 715-729,

Lamb, M. R., London, B., Pond, H. M., \& Whitt, K. A. (1998). Au- 
tomatic and controlled processes in the analysis of hierarchical structure. Psychological Science, 9, 14-19.

LAMb, M. R., Pond, H. M., \& ZAHIR, G. (2000). Contributions of automatic and controlled processes to the analysis of hierarchical structure. Journal of Experimental Psychology: Human Perception \& Performance, 26, 234-245.

Lassaline, M. E., \& Logan, G. D. (1993). Memory-based automaticity in the discrimination of visual numerosity. Journal of Experimental Psychology: Learning, Memory, \& Cognition, 19, 561-581.

Maljkovic, V., \& NaKaYAma, K. (1994). Priming of pop-out: I. Role of features. Memory \& Cognition, 22, 657-672.

Maljkovic, V., \& Nakayama, K. (1996). Priming of pop-out: II. The role of position. Perception \& Psychophysics, 58, 977-991.

MARr, D. (1982). Vision. New York; W. H. Freeman.

MARR, D., \& NishiHARA, H. K. (1978). Representation and recognition of the spatial organization of three-dimensional shapes. Proceedings of the Royal Society of London: Series B, 200, 269-294.

McCARLEY, J. S., \& He, Z. J. (2000). Asymmetry in 3-D perceptual organization: Ground-like surface superior to ceiling-like surface. Perception \& Psychophysics, 62, 540-549.

Moore, C., \& Cavanagh, P. (1998). Recovery of 3-D volume from 2tone images of novel objects. Cognition, 67, 45-71.

NaKayama, K., He, Z J., \& Shimojo, S. (1995). Visual surface representation: A critical link between lower-level and higher-level vision. In S. Kosslyn \& D. O. Osherson (Eds.), An invitation to cognitive science: Vol. 2. Visual cognition (2nd ed., pp. 1-70). Cambridge, MA: MIT Press.

Navon, D. (1977). Forest before the trees: The precedence of global features in visual perception. Cognitive Psychology, 9, 353-383.

Peterson, M. A. (1994). Object recognition processes can and do operate before figure-ground organization. Current Directions in Psychological Science, 3, 105-111.
Ramachandran, V. S. (1988). Perception of shape from shading. Nature, 331, 163-166.

Ramachandran, V. S., \& CaVanagh, P. (1985). Subjective contours capture stereopsis. Nature, 317, 527-530.

RobERTSON, L. C. (1996). Attentional persistence for features of hierarchical patterns. Journal of Experimental Psychology: General, 125, 227-249.

Robertson, L. C., Egly, R, Lamb, M. R., \& Kerth, L. (1993). Spatial attention and cuing to global and local levels of hierarchical structure. Journal of Experimental Psychology: Human Perception \& Performance, 19, 471-487.

SANOCKI, T., \& EPSTEIN, W. (1997). Priming spatial layout of scenes. Psychological Science, 8, 374-378.

SEDGWICK, H. A. (1986). Space perception. In K. R. Boff, L. Kaufman, \& J. P. Thomas (Eds.), Handbook of perception and human performance (pp. 21.1-21.57). New York: Wiley.

WARD, L. M. (1982). Determinants of attention to local and global features of visual forms. Journal of Experimental Psychology: Human Perception \& Performance, 8, 562-581.

Wertheimer, M. (1958). Principles of perceptual organization. In D. C. Beardslee \& M. Wertheimer (Eds.), Readings in perception (pp. 115135). Princeton, NJ: Van Nostrand. (Original work published 1923)

\section{NOTE}

1.The target might have changed its horizontal position within the middle row, but this was equally likely whether the orientation of the global display was reversed or not.

(Manuscript received January 25, 2000; revision accepted for publication May 18, 2000.) 